\title{
k-Parabolic Subspace Arrangements
}

\author{
Christopher Severs and Jacob White
}

School of Mathematical and Statistical Sciences, Arizona State University, Tempe, AZ 85287-1804

\begin{abstract}
In this paper, we study $k$-parabolic arrangements, a generalization of the $k$-equal arrangement for any finite real reflection group. When $k=2$, these arrangements correspond to the well-studied Coxeter arrangements. Brieskorn (1971) showed that the fundamental group of the complement of the type $W$ Coxeter arrangement (over $\mathbb{C}$ ) is isomorphic to the pure Artin group of type $W$. Khovanov (1996) gave an algebraic description for the fundamental group of the complement of the 3-equal arrangement (over $\mathbb{R}$ ). We generalize Khovanov's result to obtain an algebraic description of the fundamental group of the complement of the 3-parabolic arrangement for arbitrary finite reflection group. Our description is a real analogue to Brieskorn's description.

Résumé. Nous généralisons les arrangements k-égaux à tous les groupes de réflexions finis réels. Les arrangements ainsi obtenus sont dits k-paraboliques. Dans le cas où $\mathrm{k}=2$ nous retrouvons les arrangements de Coxeter qui sont bien connus. En 1971, Brieskorn démontra que le groupe fondamental associé au complément (complexe) de l'arrangement de Coxeter de type W est en fait isomorphe au groupe pure d'Artin de type W . En 1996, Khovanov donne une description algébrique du groupe fondamental du complément (réel) de larrangement 3-égaux. Nous généralisons le résultat de Khovanov et obtenons une description algébrique du groupe fondamental de l'espace complément d'un arrangement k-parabolique pour tous les groupes de réflexions finis et réels. Il se trouve que notre description est l'analogue réel de la description de Brieskorn.
\end{abstract}

Keywords: Subspace Arrangements, Coxeter Groups, Discrete Homotopy Theory

\section{Introduction}

A subspace arrangement $\mathscr{A}$ is a collection of linear subspaces of a finite-dimensional vector space $V$, such that there are no proper containments among the subspaces. Examples of subspace arrangements include real and complex hyperplane arrangements. One of the main questions regarding subspace arrangements is to study the structure of the complement $\mathcal{M}(\mathscr{A})=V-\cup_{X \in \mathscr{A}} X$. A combinatorial tool that has proven useful in studying the complement is the intersection lattice, $\mathcal{L}(\mathscr{A})$, which is the lattice of intersections of subspaces, ordered by inclusion. Many results regarding the homology and homotopy theory of $\mathcal{M}(\mathscr{A})$ can be found in the book by Orlik and Terao [17], when $\mathscr{A}$ is a real or complex hyperplane arrangement.

There are two interesting problems regarding homotopy of $\mathcal{M}(\mathscr{A})$ that we will concern ourselves with. The first problem is determining whether or not $\mathcal{M}(\mathscr{A})$ is an Eilenberg-MacLane space. An EilenbergMacLane space (or $K(\pi, m)$-space) is a space $X$ such that $\pi_{k}(X)=0$ for $i \neq m$ and $\pi_{m}(X)=\pi$. A $K(\pi, 1)$ subspace arrangement is an arrangement whose complement is a $K(\pi, 1)$ space. It is worth noting that not all complex hyperplane arrangements are $K(\pi, 1)$-spaces. The second problem is to find a presentation for the fundamental group of $\mathcal{M}(\mathscr{A})$. 
We will look at several motivating examples where both questions have been answered. One example of a complex $K(\pi, 1)$ hyperplane arrangement is the braid arrangement, which is the collection of "diagonals" $z_{i}=z_{j}$ for $1 \leq i<j \leq n$ from a complex $n$-dimensional vector space. In 1963, Fox and Neuwirth [14] showed that the fundamental group of the complement is isomorphic to the pure braid group. It was also shown by Fadell and Neuwirth [12] that the higher homotopy groups of the complement are trivial. Thus this is an example of a $K(\pi, 1)$-arrangement.

An example of a real $K(\pi, 1)$ subspace arrangement is the 3 -equal arrangement, which is the collection of all subspaces of the form $x_{i}=x_{j}=x_{k}$ for $1 \leq i<j<k \leq n$ in a real $n$-dimensional vector space. It was Khovanov, in 1996, who proved that this is a $K(\pi, 1)$ subspace arrangement [16]. He also gave a presentation for the fundamental group of the complement. The presentation of this group, as well as the presentation of the pure braid group, use the symmetric group in their construction. It is well known that the symmetric group is generated by adjacent transpositions $s_{i}=(i, i+1), i \in[n-1]$, subject to the following relations:

$$
\begin{aligned}
& \text { 1. } s_{i}^{2}=1 \\
& \text { 2. } s_{i} s_{j}=s_{j} s_{i} \text {, if }|i-j|>1 \\
& \text { 3. } s_{i} s_{i+1} s_{i}=s_{i+1} s_{i} s_{i+1}
\end{aligned}
$$

The braid group has presentation given by the same generating set, but subject only to relations 2 and 3. The pure braid group is the kernel of the surjective homomorphism, $\varphi$, from the braid group to the symmetric group, given by $\varphi\left(s_{i}\right)=s_{i}$ for all $i \in[n-1]$. Khovanov's presentation of the fundamental group of the complement of the 3 -equal arrangement is very similar. He defines the triplet group, which we shall denote $A_{n-1}^{\prime}$. This group has a presentation given by the generators $s_{i}$, but subject only to relations 1 and 2, and he defines the pure triplet group to be the kernel of the surjective homomorphism, $\varphi^{\prime}$ : $A_{n-1}^{\prime} \rightarrow A_{n-1}$, given by $\varphi^{\prime}\left(s_{i}\right)=s_{i}$ for all $i \in[n-1]$. Khovanov showed that the fundamental group of the complement of the 3-equal arrangement is isomorphic to the pure triplet group. Thus, Khovanov found a "real analogue" to the results of Fadell, Fox and Neuwirth.

The work of Fadell and Neuwirth has been generalized to other hyperplane arrangements. A simplicial hyperplane arrangement is an arrangement whose regions are simplicial cones. In 1972, Deligne [11] showed that the complexification of any simplicial hyperplane arrangement is a $K(\pi, 1)$-arrangement. Given a finite real reflection group $W$, consider the complexification of the reflection arrangement associated to $W$. Since reflection arrangements are simplicial, their complexifications are $K(\pi, 1)$-arrangements. Moreover, in 1971 Brieskorn [9] found that the fundamental group of the complement is isomorphic to the pure Artin group of type $W$. We review the definition of Artin groups in Section 4.

Our primary interest is to give "real analogues" of these results for subspace arrangements in $\mathbb{R}^{n}$ that correspond to finite real reflection groups. In particular, given a finite real reflection group $W$, we define a family of (real) subspace arrangements which we call $k$-parabolic arrangements. We show in Theorem 4.1 that the fundamental group of the complement of a 3-parabolic arrangement has the following simple description. We construct a new Coxeter group $W^{\prime}$ on the same generating set $S$ as $W$, but we relax all relations of $W$ that are not commutative relations nor involutions. Then the fundamental group is the kernel of a surjective homomorphism $\varphi^{\prime}: W^{\prime} \rightarrow W$ given by $\varphi^{\prime}(s)=s$ for all $s \in S$. It turns out that the 3-parabolic arrangement is also a $K(\pi, 1)$ arrangement, a result due to Davis et al. (Theorem 0.1.9 in [10]). 
Our primary tool for finding our presentation is the notion of discrete homotopy theory. Discrete homotopy theory is a theory that was developed in [2]. The theory involves constructing a bigraded sequence of groups defined on an abstract simplicial complex that are invariants of a combinatorial nature. Instead of being defined on the topological space of a geometric realization of a simplicial complex, the discrete homotopy groups are defined in terms of the combinatorial connectivity of the complex. That is, we are interested in how simplices intersect. In this paper, we show that the discrete fundamental group of the Coxeter complex is isomorphic to $\pi_{1}$ of the complement of the 3-parabolic arrangement. Thus, our result shows that sometimes we can replace a group defined in terms of the topology of the space with a group defined in terms of the combinatorial structure of the space.

In Section 2 we give a definition of the $k$-parabolic arrangement, and review some necessary definitions related to Coxeter groups. We also relate $k$-parabolic arrangements to previous analogues of the $k$-equal arrangement given by Björner and Sagan [7] for types $B$ and $D$. In Section 3, we give a brief overview of discrete homotopy theory and the definition of the Coxeter complex. Then we give an isomorphism between the classical fundamental group of the complement of the 3-parabolic arrangement and the discrete fundamental group of the corresponding Coxeter complex. In Section 4, we use this isomorphism and a study of discrete homotopy loops in the Coxeter complex to obtain our algebraic description of the fundamental group of the complement of the 3-parabolic arrangement. In Section 5 we conclude with some open questions related to $\mathscr{W}_{n, k}$-arrangements as well as a discussion on the $K(\pi, 1)$ problem.

\section{Definition of the $\mathcal{W}_{n, k}$-arrangement}

Let $W$ be a finite real reflection group acting on $\mathbb{R}^{n}$ and fix a root system $\Phi$ associated to $W$. Let $\Pi \subset \Phi$ be a fixed simple system. Finally, let $S$ be the set of simple reflections associated to $\Pi$. Assume that $\Pi$ spans $\mathbb{R}^{n}$. We let $m(s, t)$ denote the order of $s t$ in $W$. We know that $m(s, s)=1$ and $m(s, t)=m(t, s)$ for all $s, t \in S$. Finally, given a root $\alpha$, let $s_{\alpha}$ denote the corresponding reflection, and let $(\cdot, \cdot)$ denote the standard inner product.

Recall that there is a hyperplane arrangement associated to $W$, called the Coxeter arrangement $\mathscr{H}(W)$, which consists of hyperplanes $H_{\alpha}=\left\{x \in \mathbb{R}^{n}:(x, \alpha)=0\right\}$ for each $\alpha \in \Phi^{+}$. Since $\Pi$ spans $\mathbb{R}^{n}$, the Coxeter arrangement is central and essential, which implies that the intersection of all the hyperplanes is the origin.

Since we are generalizing the $k$-equal arrangement, which corresponds to the case $W=A_{n}$, we use it as our motivation. For this paper, we will actually work with the essentialized $k$-equal arrangement. The $k$-equal arrangement, $\mathscr{A}_{n, k}$, is the collection of all subspaces given by $x_{i_{1}}=x_{i_{2}}=\ldots=x_{i_{k}}$ over all indices $\left\{i_{1}, \ldots, i_{k}\right\} \subset[n+1]$, with the relation $\sum_{1}^{n+1} x_{i}=0$. The $k$-equal arrangement is an arrangement that has been studied extensively ([6], [8], [16]). We note that the intersection poset $\mathcal{L}\left(\mathscr{A}_{n, k}\right)$ is a subposet of $\mathcal{L}\left(\mathscr{H}\left(A_{n}\right)\right)$. There is already a well-known combinatorial description of both of these posets. The poset of all set partitions of $[n+1]$ ordered by refinement is isomorphic to $\mathcal{L}\left(\mathscr{H}\left(A_{n}\right)\right)$, and under this isomorphism, $\mathcal{L}\left(\mathscr{A}_{n, k}\right)$ is the subposet of set partitions where each block is either a singleton, or has size at least $k$. However, our generalization relies on a lesser-known description of these posets in terms of parabolic subgroups.

Definition 2.1 A subgroup $G \subseteq W$ is a parabolic subgroup if there exists a subset $T \subseteq S$ of simple reflections, and an element $w \in W$ such that $G=\left\langle w T w^{-1}>\right.$. If $w$ can be taken to be the identity, then $G$ is a standard parabolic subgroup. We view $\left(G, w T w^{-1}\right)$ as a Coxeter system, and call $G$ irreducible if $\left(G, w T w^{-1}\right)$ is an irreducible system. 
It is well known that the lattice of standard parabolic subgroups, ordered by inclusion, is isomorphic to the Boolean lattice. However, the lattice of all parabolic subgroups, $\mathscr{P}(W)$, ordered by inclusion, was shown by Barcelo and Ihrig [1] to be isomorphic to $\mathcal{L}(\mathscr{H}(W))$. Since this isomorphism is essential to our generalization, we review it. The isomorphism is given by sending a parabolic subgroup $G$ to $F i x(G)=\left\{x \in \mathbb{R}^{n}: w x=x, \forall w \in G\right\}$, and the inverse is given by sending an intersection of hyperplanes $X$ to $\operatorname{Gal}(X)=\{w \in W: w x=x, \forall x \in X\}$.

This Galois correspondence gives a description of $\mathcal{L}\left(\mathscr{H}\left(A_{n}\right)\right)$ in terms of parabolic subgroups of $A_{n}$. We also obtain another description of $\mathcal{L}\left(\mathscr{A}_{n, k}\right)$ under this correspondence.

Proposition 2.2 The Galois correspondence gives a bijection between subspaces of $\mathscr{A}_{n, k}$ and irreducible parabolic subgroups of $A_{n}$ of rank $k-1$.

Proof: Let $X$ be a subspace of $\mathbb{R}^{n+1}$ given by $x_{1}=\ldots=x_{k}$. The $k$-equal arrangement is the orbit of $X$ under the action of $A_{n}=S_{n+1}$, and $\operatorname{Gal}(X)=<(1,2), \ldots,(k-1, k)>$, hence is irreducible. For $w \in A_{n}, \operatorname{Gal}(w X)=w \operatorname{Gal}(X) w^{-1}$, so all of the subspaces in the $k$-equal arrangement have irreducible Galois groups.

Conversely, every irreducible parabolic subgroup of rank $k-1$ in $A_{n}$ is the Galois group of some subspace in the $k$-equal arrangement. To see this, consider an irreducible parabolic subgroup $G$ of rank $k-1$. Then there exists a standard parabolic subgroup $H$ and an element $w \in W$ such that $G=w H w^{-1}$. Since $H$ is an irreducible standard parabolic subgroup, $H=<(i, i+1), \ldots,(i+k-1, i+k)>$ for some $1 \leq i \leq n+1-k$. Thus, $\operatorname{Fix}(H)$ is given by $x_{i}=\ldots=x_{k}$, and $\operatorname{Fix}(G)=F i x\left(w H w^{-1}\right)=w F i x(G)$ is given by $x_{w(i)}=\ldots x_{w(k)}$, which is a subspace in the $k$-equal arrangement.

With this proposition as motivation, we give the following definition for a $k$-parabolic arrangement.

Definition 2.3 Let $W$ be an finite real reflection group of rank $n$. Let $\mathscr{P}_{n, k}(W)$ be the collection of all irreducible parabolic subgroups of $W$ of rank $k-1$.

Then the $k$-parabolic arrangement $\mathscr{W}_{n, k}$ is the collection of subspaces

$$
\left\{F i x(G): G \in \mathscr{P}_{n, k}(W)\right\} .
$$

The $k$-parabolic arrangements have many properties in common with the $k$-equal arrangements. Both of these arrangements can be embedded in the corresponding Coxeter arrangement. That is, every subspace in these arrangements can be given by intersections of hyperplanes of the Coxeter arrangements. Moreover, $\mathcal{L}\left(\mathscr{W}_{n, k}\right)$ is a subposet of $\mathcal{L}(\mathscr{H}(W))=\mathcal{L}\left(\mathscr{W}_{n, 2}\right)$, and these arrangements are invariant under the action of $W$. Indeed, consider a subspace $X$ in $\mathscr{W}_{n, k}$ and an element $w \in W$. Since $X$ is in $\mathscr{W}_{n, k}$, $\operatorname{Gal}(X)$ is an irreducble parabolic subgroup of rank $k-1$. It is clear that $\operatorname{Gal}(w X)=w \operatorname{Gal}(X) w^{-1}$, so $\operatorname{Gal}(w X)$ is also an irreducible parabolic subgroup of rank $k-1$, whence $\operatorname{Gal}(w X) \in \mathscr{P}_{n, k}(W)$. Since $\operatorname{Fix}(\operatorname{Gal}(w X))=w X$, it follows that $w X \in \mathcal{W}_{n, k}$.

When $W$ is of type $A$, we see that we have recovered the $k$-equal arrangement. To see what happens when $W$ is type $B$ or $D$, first we recall type $B$ and $D$ analogues of the $k$-equal arrangement. In 1996, Björner and Sagan defined a class of subspace arrangements of type $B$ and $D$ [7], which they call the $\mathscr{B}_{n, k, h}$-arrangements and $\mathscr{D}_{n, k}$-arrangements.

Definition 2.4 The $\mathscr{D}_{n, k}$-arrangement consists of subspaces given by $\pm x_{i_{1}}= \pm x_{i_{2}}=\ldots= \pm x_{i_{k}}$, over distinct indices $i_{1}, \ldots, i_{k}$. The $\mathscr{B}_{n, k, h}$-arrangements are obtained from the $\mathscr{D}_{n, k}$-arrangements by including subspaces given by $x_{i_{1}}=\ldots=x_{i_{h}}=0$ over distinct indices $i_{1}, \ldots, i_{h}$, with $h<k$. 
The Betti numbers of $\mathcal{M}\left(\mathscr{B}_{n, k, h}\right)$ were computed by Björner and Sagan in [7], while the Betti numbers of $\mathcal{M}\left(\mathscr{D}_{n, k}\right)$ were computed by Kozlov and Feichtner in [13].

Example 2.5 (When $W$ is of type $B$ ) When $W$ is of type $B$, the $k$-parabolic arrangement is the $\mathscr{B}_{n, k, k-1 \text { - }}$ arrangement of Björner and Sagan [7]. Recall that $B_{n}$ has presentation given by generators $s_{i}, 0 \leq i \leq$ $n$, such that $\left\langle s_{1}, \ldots, s_{n}>\right.$ generate the symmetric group, $\left(s_{0} s_{1}\right)^{4}=1$, and $s_{0} s_{i}=s_{i} s_{0}$ for $i>1$. It is well-known that the $\mathscr{B}_{n, k, k-1}$-arrangement is the orbit of two subspaces given by $x_{1}=\ldots=x_{k}$ and $x_{1}=\ldots=x_{k-1}=0$, under the action of $B_{n}$. Clearly the Galois groups of these two spaces are given by $\left\langle s_{1}, \ldots, s_{k-1}>\right.$ and $<s_{0}, \ldots, s_{k-2}>$. These are both irreducible parabolic subgroups of rank

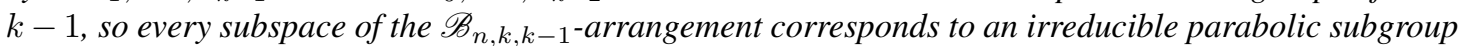
of rank $k-1$. Similarly, given an irreducible parabolic subgroup of rank $k-1$, it is not hard to show that this subgroup corresponds to a subspace in the $\mathscr{B}_{n, k, k-1}$-arrangement. The argument is similar to the case for type $A$, and we omit the details.

\section{Discrete Homotopy Theory}

To facilitate the proofs of our algebraic description for $\pi_{1}\left(\mathcal{M}\left(\mathscr{W}_{n, k}\right)\right)$, first we give a combinatorial description of $\pi_{1}\left(\mathcal{M}\left(\mathscr{W}_{n, k}\right)\right)$ in terms of discrete homotopy theory of the Coxeter complex for $W$. As motivation, we mention the following result:

Theorem 3.1 Let $\mathcal{M}\left(\mathscr{A}_{n, k}\right)$ be the complement of the k-equal arrangement $\mathscr{A}_{n, k}$. Let $\mathscr{C}\left(A_{n}\right)$ be the order complex of the Boolean lattice.

Then $\pi_{1}\left(\mathcal{M}\left(\mathscr{A}_{n, k}\right)\right) \cong A_{1}^{n-k+1}\left(\mathscr{C}\left(A_{n}\right)\right)$, where $A_{1}^{q}$ is a discrete homotopy group, to be defined below.

This result was shown independently by Björner [5] and Babson (appears in [3]) in 2001). It turns out that the order complex of the Boolean lattice is the Coxeter complex of type $A$, which explains our choice of notation.

One of the original motivations for discrete homotopy theory was to create a sequence of groups for studying social networks being modeled as simplicial complexes. However, as Theorem 3.1 shows, discrete homotopy theory has applications in other areas of mathematics. We will show that there is an isomorphism between $\pi_{1}\left(\mathcal{M}\left(\mathscr{W}_{n, k}\right)\right)$ and the discrete fundamental group, $A_{1}^{n-k+1}$, of the Coxeter complex, a combinatorial structure associated to the Coxeter arrangement. Essentially, we are replacing a topologically defined group with a combinatorially defined group. First, however, we give an overview of some of the needed basic definitions and results from discrete homotopy theory. Many details and background history of discrete homotopy theory can be found in [2].

Fix a positive integer $d$. Let $\Delta$ be a simplicial complex of dimension $d$, fix $0 \leq q \leq d$, and let $\sigma_{0} \in \Delta$ be maximal with dimension $\geq q$. Two simplicies $\sigma$ and $\tau$ are $q$-near if they share $q+1$ elements. A $q$-chain is a sequence $\sigma_{1}, \ldots, \sigma_{k}$, such that $\sigma_{i}, \sigma_{i+1}$ are $q$-near for all $i$. A $q$-loop based at $\sigma_{0}$ is a $q$-chain with $\sigma_{1}=\sigma_{k}=\sigma_{0}$.

Definition 3.2 We define an equivalence relation, $\simeq$ on $q$-loops with the following conditions:

1. The q-loop

$$
(\sigma)=\left(\sigma_{0}, \sigma_{1}, \ldots, \sigma_{i}, \sigma_{i+1}, \ldots, \sigma_{n}, \sigma_{0}\right)
$$

is equivalent to the q-loop

$$
(\sigma)^{\prime}=\left(\sigma_{0}, \sigma_{1}, \ldots, \sigma_{i}, \sigma_{i}, \sigma_{i+1}, \ldots, \sigma_{n}, \sigma_{0}\right),
$$




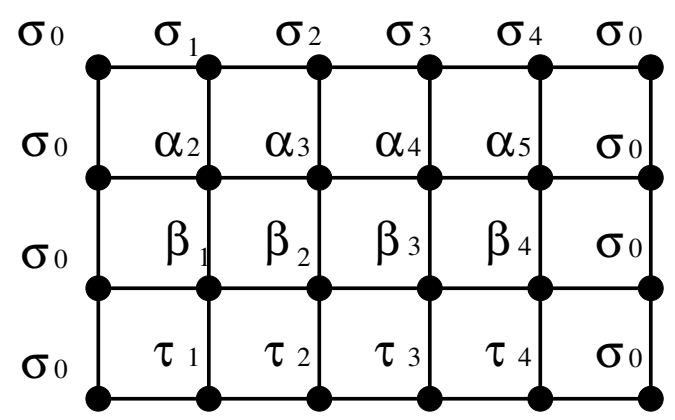

Fig. 1: An example of a homotopy grid

which we refer to as stretching.

2. If $(\sigma)$ and $(\tau)$ are two q-loops that have the same length then they are equivalent if there is a diagram as in figure 1. The vertices represent simplices, and two vertices are connected by an edge if and only if the corresponding simplices are q-near. Thus, every row is a q-loop based at $\sigma_{0}$, and every column is a q-chain. Such a diagram is called a (discrete) homotopy between $(\sigma)$ and $(\tau)$.

Define $A_{1}^{q}\left(\Delta, \sigma_{0}\right)$ to be the collection of equivalence classes of $q$-loops based at $\sigma_{0}$. Then the operation of concatenation of $q$-loops gives a group operation on $A_{1}^{q}\left(\Delta, \sigma_{0}\right)$, the discrete homotopy group of $\Delta$. The identity is the equivalence class containing the trivial loop $\left(\sigma_{0}\right)$, and given an equivalence class $[\sigma]$ for the $q$-loop $(\sigma)=\left(\sigma_{0}, \sigma_{1}, \ldots, \sigma_{k}, \sigma_{0}\right)$, the inverse $[\sigma]^{-1}$ is the equivalence class of $\left(\sigma_{0}, \sigma_{k}, \sigma_{k-1}, \ldots, \sigma_{2}, \sigma_{1}, \sigma_{0}\right)$. As in classical topology, if a pair of maximal simplices $\sigma, \tau$ of dimension at least $q$ in $\Delta$ are $q$-connected, then $A_{1}^{q}(\Delta, \sigma) \cong A_{1}^{q}(\Delta, \tau)$. Thus, in the case $\Delta$ is $q$-connected, we will set $A_{1}^{q}(\Delta)=A_{1}^{q}\left(\Delta, \sigma_{0}\right)$ for any maximal simplex $\sigma_{0} \in \Delta$ of dimension at least q.

Before we use discrete homotopy theory, we need a result from [2] that relates discrete homotopy theory of a simplicial complex to classical homotopy theory of a related space. Given $0 \leq q \leq d$, let $\Gamma^{q}(\Delta)$ be a graph whose vertices are maximal simplices of $\Delta$ of size at least $q$, and with edges between two simplices $\sigma, \tau$, if and only if $\sigma$ and $\tau$ are $q$-near. Then the following result relates $A_{1}^{q}\left(\Delta, \sigma_{0}\right)$ in terms of a cell complex related to $\Gamma^{q}(\Delta)$.

Proposition 3.3 (Proposition 5.12 in [2])

$$
A_{1}^{q}\left(\Delta, \sigma_{0}\right) \cong \pi_{1}\left(X_{\Gamma}, \sigma_{0}\right)
$$

where $X_{\Gamma}$ is a cell complex obtained by gluing a 2-cell on each 3 - and 4-cycle of $\Gamma=\Gamma^{q}(\Delta)$.

Let $W, \mathcal{H}(W), \Phi, \Pi, S$ be as in section 2. As mentioned previously, we study the discrete homotopy groups of the Coxeter complex associated to $W$, and relate them to $\pi_{1}\left(\mathcal{M}\left(\mathscr{W}_{n, k}\right)\right)$. The majority of these details can be found in Section 1.14 in Humphrey's book on Coxeter groups [15]. The concepts regarding fans and zonotopes can be found in Chapter 7 of Zeigler's book on polytopes [18].

For a given set $I \subseteq S$, let $W_{I}=<I>$, and $\Pi_{I}=\left\{\alpha \in \Pi: s_{\alpha} \in I\right\}$. We can associate to $W_{I}$ the set of points $C_{I}=\left\{x \in \mathbb{R}^{n}:(x, \alpha)=0, \forall \alpha \in \Pi_{I}\right.$, and $\left.(x, \alpha)>0, \forall \alpha \in \Pi-\Pi_{I}\right\}$. The set $C_{I}$ is the intersection of hyperplanes $H_{\alpha}$ for $\alpha \in \Pi_{I}$ with certain open half-spaces. We see that $C_{\emptyset}$ corresponds to the interior of a fundamental region, and $C_{S}$ is the origin. 
For a given coset $w W_{I}$, we can associate the set of points $w C_{I}$. The collection $\mathscr{C}(W)$ of $w C_{I}$ for all $w \in W$, and all $I \subseteq S$ partitions $\mathbb{R}^{n}$, and is called the Coxeter complex of $W$. The face poset of the Coxeter complex can be viewed as the collection of cosets $w W_{I}$ for any $w \in W, I \subseteq S$, ordered by reverse inclusion. We note that this poset is not $\mathcal{L}(\mathscr{H}(W))$. For $W=A_{n}$, we have already mentioned that $\mathcal{L}(\mathscr{H}(W))$ is isomorphic to the partition lattice. The face poset of the braid arrangement, however, is isomorphic to the order complex of the boolean lattice. Since chains in the boolean lattice are in oneto-one correspondence with ordered set partitions, these two posets are related, but are very different.

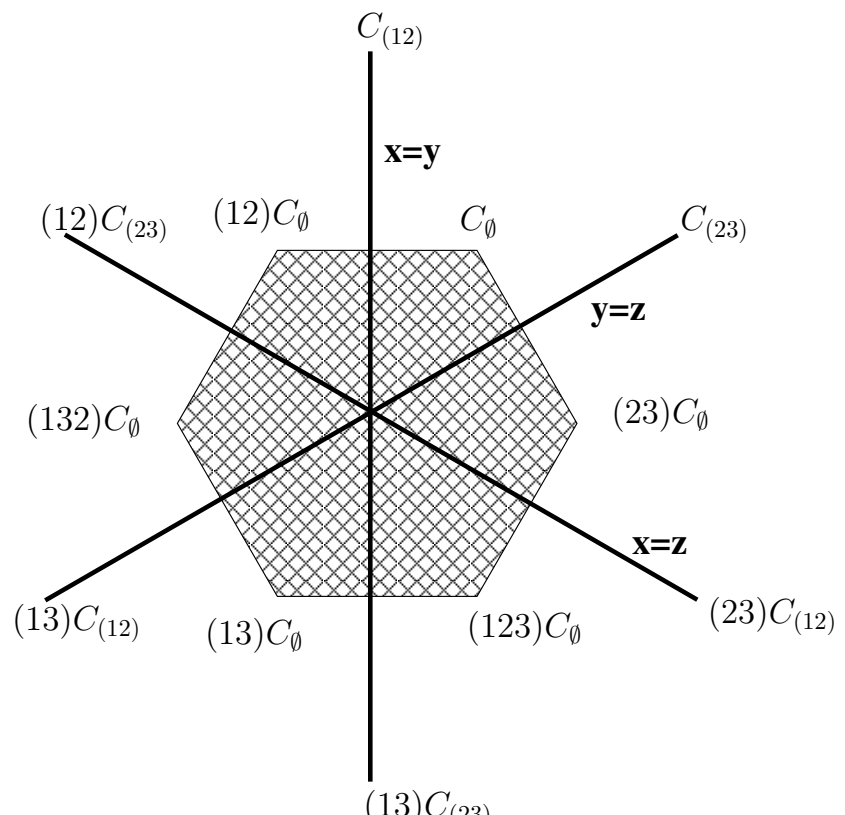

Fig. 2: Coxeter Complex and Zonotope for $W=A_{3}$. Note that $C_{S}$ is the origin.

For a given $w, I$, the closure of $w C_{I}$ is a convex polyhedral cone. In fact, the collection of all $w \bar{C}_{I}$ forms a fan of $\mathbb{R}^{n}$, which is the fan associated to $\mathscr{H}(W)$. Under this view, the sets $w \bar{C}_{I}$ are the faces of the arrangement.

Recall that we can associate a zonotope to a hyperplane arrangement. That is, given line segments of unit length normal to the hyperplanes, one can form a polytope by taking the Minkowski sum of these line segments. For a Coxeter arrangement of type $W$ this zonotope is called the $W$-Permutahedron. Also, the fan of the arrangement is the normal fan of the zonotope. Thus, we can label the faces of the $W$-Permutahedron by cosets $w W_{I}$, where a face $F$ gets the label $w W_{I}$ if the normal cone for $F$ is $w \bar{C}_{I}$. Under this labeling, the face poset of the $W$-Permutahedron is indexed by cosets $w W_{I}$ for all $w \in W, I \subseteq S$, ordered by inclusion.

We observe that in the $W$-Permutahedron, the vertices correspond to elements of $W$, and two vertices share an edge if and only if the corresponding regions share an $(n-1)$-dimensional boundary, that is if and only if the corresponding elements of $W$ differ by multiplication on the right by a simple reflection. 
From this it follows that the graph (one-skeleton) of the $W$-Permutahedron is the graph $\Gamma^{n-2}(\mathscr{C}(W))$ defined before Proposition 3.3

We also characterize the cycles that are boundaries of 2-faces in the $W$-Permutahedron. Given a 2dimensional face $F$ and a vertex $w$ in $F$, we see that one edge adjacent to $w$ in $F$ is of the form $w, w s$ for some $s \in S$. Likewise, one of the two edges of $F$ incident to the edge $w, w s$ is the edge $w s, w s t$, where $t \in S-s$. Thus we see that the coset associated to the normal cone of $F$ contains both $w W_{s}$ and $w s W_{t}$. Likewise, it is the smallest coset to contain these two cosets, so the corresponding coset is given by $w W_{\{s, t\}}$. The cycle that is the boundary of $F$ is seen to have length $2 m(s, t)$. This means that the graph has no 3-cycles, and 4-cycles are boundaries of faces which correspond to a coset of $W_{\{s, t\}}$, where $s, t \in S$ and $m(s, t)=2$. The fact that the graph has no 3 -cycles will turn out to be useful in section 4 .

Now we turn to the main result of this section.

Theorem 3.4 Let $\mathcal{M}\left(\mathscr{W}_{n, k}\right)$ be the complement of the k-parabolic arrangement $\mathscr{W}_{n, k}$.

Then $\pi_{1}\left(\mathcal{M}\left(\mathscr{W}_{n, k}\right)\right) \cong A_{1}^{n-k+1}(\mathscr{C}(W))$.

The proof is given in the full version of the paper [4].

\section{An algebraic description of $\pi_{1}\left(\mathcal{M}\left(\mathscr{W}_{n, 3}\right)\right)$}

In this section, we give a description of $\pi_{1}\left(\mathcal{M}\left(\mathscr{W}_{n, k}\right)\right)$ that is similar to the idea of a pure Artin group. In our case, the group we consider is a (possibly infinite) Coxeter group. Recall that $W$ affords the following presentation: $W$ is generated by $S$ subject to the relations:

1. $s^{2}=1, \forall s \in S$

2. $s t=t s, \forall s, t \in S$ such that $m(s, t)=2$

3. $s t s=t s t, \forall s, t \in S$, such that $m(s, t)=3$

i. $\underbrace{s t s t \cdots}_{i}=\underbrace{t s t s \cdots}_{i}, \forall s, t \in S$, such that $m(s, t)=i$

where of course we have no relation of the form $s t \cdots=t s \cdots$ if $m(s, t)=\infty$.

If $G$ is a group generated by $S$ subject to every relation except relations of type 1, then $G$ is an Artin group. There is a surjective homomorphism $\varphi: G \rightarrow W$ given by $\varphi(s)=s$ for all $s \in S$. The kernel of $\varphi$ is the pure Artin group. As stated in the introduction, the pure Artin group is isomorphic to the fundamental group of the complement of the complexification of the Coxeter arrangement for $W$. The goal of this section is to give a real analogue of this result for the $\mathscr{W}_{n, 3}$-arrangements.

In our case, let $W^{\prime}$ be a group on $S$ subject to only the relations of type 1 and 2. Equivalently, $W^{\prime}$ is subject to $s^{2}=1$ for all $s \in S$, and two elements $s, t \in S$ commute in $W^{\prime}$ if and only if they commute in $W$. In essence, given the Dynkin diagram $D$ for $W, W^{\prime}$ is obtained by replacing all the edge labels in $D$ with the edge label $\infty$, and letting $W^{\prime}$ be the resulting Coxeter group.

Consider the surjective homomorphism $\varphi^{\prime}: W^{\prime} \rightarrow W$ given by $\varphi^{\prime}(s)=s$ for all $s \in S$. Then the following result holds: 


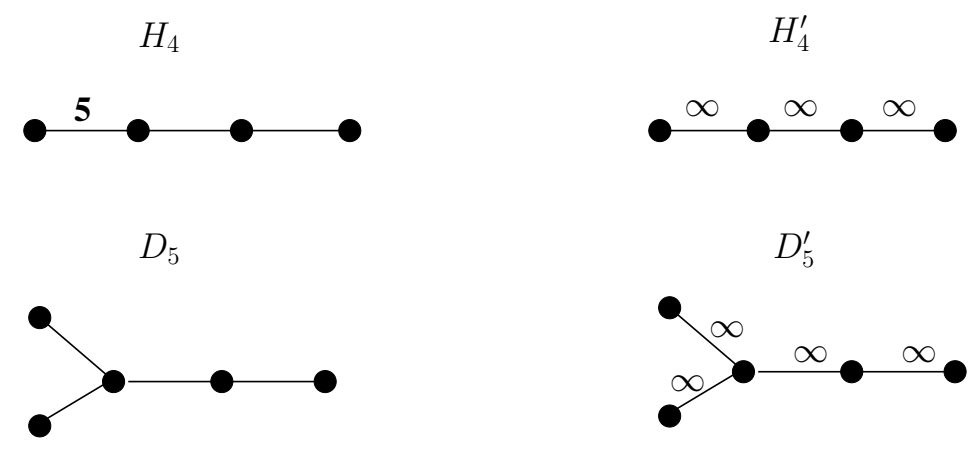

Fig. 3: Dynkin diagrams for $W$ and $W^{\prime}$

Theorem 4.1 $\pi_{1}\left(\mathcal{M}\left(\mathscr{W}_{n, 3}\right)\right) \cong \operatorname{ker} \varphi^{\prime}$.

When $W$ is of type $A$ or $B$, Theorem 4.1 was shown by Khovanov [16], where the arrangements are referred to using different terminology. However, we give a proof for any finite real reflection group.

As a result of Theorem 3.4, we know that we can study $\pi_{1}\left(\mathcal{M}\left(\mathscr{W}_{n, k}\right)\right)$ using discrete homotopy theory of $\mathscr{C}(W)$. Before we prove Theorem 4.1 we first investigate the structure of $(n-2)$-loops in $\mathscr{C}(W)$ in more detail. For the duration of the section we will use the term loop to mean $(n-2)$-loop. To any such loop $(\sigma)=\left(\sigma_{0}, \ldots, \sigma_{\ell}, \sigma_{0}\right)$ in $\mathscr{C}(W)$ we associate a sequence of elements of $S \cup\{1\}$ of length $\ell$ in the following way: For any $i \in[\ell]$, if $\sigma_{i}=\sigma_{i-1}$, let $s_{i}=1$. Otherwise let $s_{i}$ be the unique element $s$ of $S$ for which $\sigma_{i-1} s=\sigma_{i}$. Thus we associate a word $f(\sigma)$ in $S^{*}$ to $(\sigma)$ : the product of the elements of the corresponding sequence in order.

We note that if $(\sigma)$ is a loop, then $f(\sigma)=1$ in $W$. This implies that when viewing $f(\sigma)$ as a product in $W^{\prime}, f(\sigma) \in \operatorname{ker} \varphi^{\prime}$. We also note that to any element $w=s_{1} \cdots s_{k}$ in $S^{*}$ we can associate a chain $g(w)=\left(\sigma_{0}, \sigma_{0} s_{1}, \ldots, \sigma_{0} s_{1} \cdots s_{k}\right)$, where the elements $s_{1} \cdots s_{i}$ are being viewed as elements of $W$. If $w=1$ when viewed as an element of $W$, then $g(w)$ is actually a loop. It is easy to see that for two loops $(\sigma),(\tau), f((\sigma) *(\tau))=f(\sigma) f(\tau)$, and if $u, v \in S^{*}, u=v=1$ in $W$, then $g(u v)=g(u) * g(v)$.

Suppose there is a homotopy between two loops $(\sigma)$ and $(\tau)$ of the same length. Since $\Gamma^{n-2}(\mathcal{C}(W))$ does not have any 3-cycles, it turns out that there is a (discrete) homotopy between them where adjacent rows in the grid follow one of the three following discrete homotopy operations. In each case, we also show how the associated words differ between the adjacent rows. Finally, $e$ refers to the identity element of $W$.

(T1) Repeating simplices. A simplex $\alpha$ is repeated consecutively on the top row, and a different simplex $\beta$ is repeated consecutively on the bottom row. Note that this results in no change in the associated words.

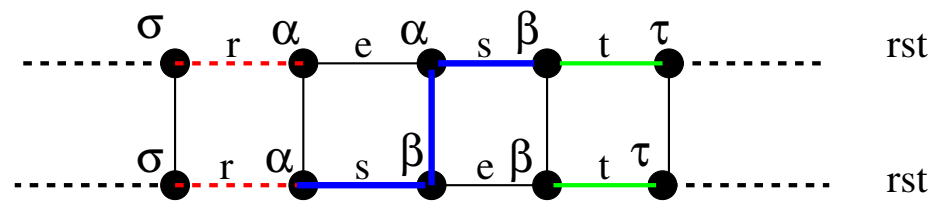

(T2) Inserting or removing a simplex. On one row there are three adjacent identical simplices $\alpha$, and on 
the bottom row the middle simplex of this triple is replaced with a new simplex $\beta$ that is $(n-2)$-near $\alpha$. Note that the corresponding words differ by an involution relation.

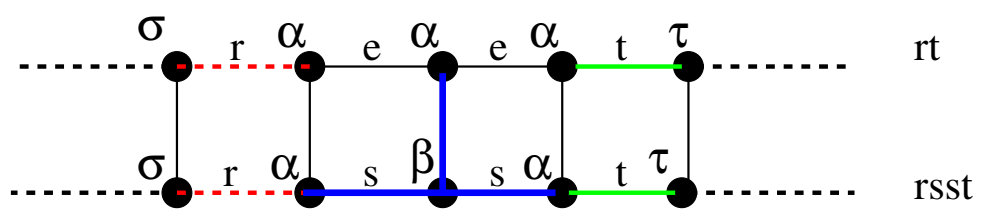

(T3) Exchanging pairs that are $(n-2)$-near. We happen to know that $(\alpha, \beta, \tau, \gamma)$ is a loop of distinct simplices. We construct a discrete homotopy as shown in the figure. We note that the resulting words differ by an application of a commutative relation. It is also worth noting this operation can only be performed when $s, t$ commute.

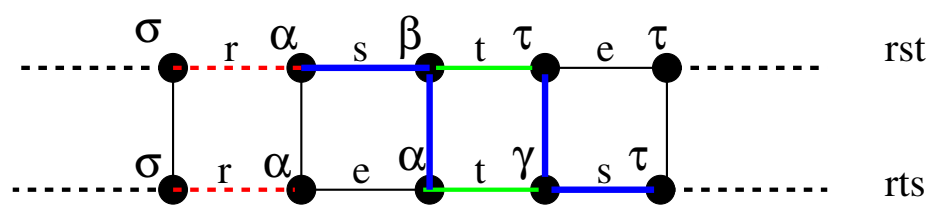

Thus for any discrete homotopy operation, the corresponding words are either equal, or differ by one of the generating relations of $W^{\prime}$. In the full paper [4], we use this observation to prove the following lemma.

Lemma 4.2 1. Let $(\sigma),(\tau)$ be loops. If $(\sigma) \simeq(\tau)$ then $f(\sigma)=f(\tau)$ in $W^{\prime}$.

2. Let $w \in S^{*}$. If $w=1$ in $W^{\prime}$, then $g(w)$ is contractible.

3. Let $w, v \in S^{*}$. If $w=v$ in $W^{\prime}$, then $g(w) \simeq g(v)$.

\section{Proof of Theorem 4.1;}

The isomorphism is given by sending the equivalence class with representative $(\sigma)$ to $f(\sigma)$, and the inverse is given by sending an element $w \in W^{\prime}$, expressed as $s_{1} s_{2} \cdots s_{k}, k \in \mathbb{N}, s_{1}, \ldots, s_{k} \in S$, to $g\left(s_{1} \cdots s_{k}\right)$. The details that these functions are well-defined isomorphisms is given in the full paper [4].

\section{Conclusion and Open Problems}

It follows as a result of Corollary 5 in [8] that for $k>3$, the $k$-parabolic arrangements are not $K(\pi, 1)$. However, the $\mathscr{W}_{n, 3}$-arrangement is a $K(\pi, 1)$-arrangement. As a result of Davis, Januszkiewicz and Scott, if $\mathscr{A}$ is any collection of codimension 2 subspaces of $\mathscr{H}(W)$ that are invariant under the action of $W$, then $\mathscr{A}$ is a $K(\pi, 1)$-arrangement (Theorem 0.1.9 in [10]).

Currently there is no presentation for the fundamental groups of the complement of such $W$-invariant arrangements. Motivated by our results, and the work of Khovanov [16], we give the following conjectured presentation. 
Conjecture 5.1 Let $\mathscr{P}$ be a collection of rank 2 parabolic subgroups of a finite real reflection group $W$ such that $\mathscr{P}$ is closed under conjugation, and let $\mathscr{W}=\{F i x(G): G \in \mathscr{P}\}$ Define a new Coxeter group $W^{\prime}$ with the same generating set $S$ as $W$, and subject to:

$m^{\prime}(s, t)= \begin{cases}\infty & \text { if }<s, t>\in \mathscr{P} \\ m(s, t) & \text { else }\end{cases}$
and let $\varphi: W^{\prime} \rightarrow W$ be given by sending $s \rightarrow$ sfor all $s \in S$. Then $\pi_{1}(\mathcal{M}(\mathscr{W})) \cong \operatorname{ker} \varphi$.

In [2], a definition is given for higher discrete homotopy groups, which are denote $A_{m}^{q}\left(\Delta, \sigma_{0}\right)$. A natural question is whether or not these groups are related to the higher homotopy groups of $\mathcal{M}\left(\mathscr{W}_{n, k}\right)$.

Conjecture 5.2 Let $\mathcal{M}\left(\mathscr{W}_{n, k}\right)$ be the complement of the $k$-parabolic arrangement $\mathscr{W}_{n, k}$.

Then $\pi_{m}\left(\mathcal{M}\left(\mathscr{W}_{n, k}\right)\right) \cong A_{m}^{n-k+1}(\mathscr{C}(W))$.

For $m<k$, it would suffice to show that $A_{m}^{n-k+1}(\mathscr{C}(W))$ is trivial. The conjecture becomes interesting for $k>3, m=k$, because in this case the $k$-th homology group of $\mathcal{M}\left(\mathscr{W}_{n, k}\right)$ is isomorphic to the $k$-th homotopy group. Thus, one could find the formulas for the first non-zero Betti numbers using discrete homotopy theory. Determining the Betti numbers for the $k$-parabolic arrangements is also an open problem, in the case that $W$ is an exceptional groups.

Finally, one may if it is possible to generalize Theorem 3.4 to other hyperplane arrangements. That is, given a hyperplane arrangement $\mathscr{H}$, let $\mathscr{C}(\mathscr{H})$ be the face complex of $\mathscr{H}$. Is there a subspace arrangement $\mathscr{A}$ for which $\pi_{1}(\mathcal{M}(\mathscr{A})) \cong A_{1}^{n-2}(\mathscr{C}(\mathscr{H}))$ ? This would be an example of using discrete homotopy theory of a complex that arises from geometry to study a topological space related to the original complex.

\section{References}

[1] Hélène Barcelo and Edwin Ihrig, Lattices of parabolic subgroups in connection with hyperplane arrangements, J. Algebraic Combin. 9 (1999), no. 1, 5-24. MR MR1676736 (2000g:52023)

[2] Hélène Barcelo, Xenia Kramer, Reinhard Laubenbacher, and Christopher Weaver, Foundations of a connectivity theory for simplicial complexes, Adv. in Appl. Math. 26 (2001), no. 2, 97-128. MR MR1808443 (2001k:57029)

[3] Hélène Barcelo and Reinhard Laubenbacher, Perspectives on A-homotopy theory and its applications, Discrete Math. 298 (2005), no. 1-3, 39-61. MR MR2163440 (2006f:52017)

[4] Hélène Barcelo, Christopher Severs, and Jacob White, k-parabolic subspace arrangements, 2009.

[5] Anders Bjorner, Personal communication, 1999.

[6] Anders Björner and László Lovász, Linear decision trees, subspace arrangements and Möbius functions, J. Amer. Math. Soc. 7 (1994), no. 3, 677-706. MR MR1243770 (95e:52024)

[7] Anders Björner and Bruce E. Sagan, Subspace arrangements of type $B_{n}$ and $D_{n}$, J. Algebraic Combin. 5 (1996), no. 4, 291-314. MR MR1406454 (97g:52028)

[8] Anders Björner and Volkmar Welker, The homology of " $k$-equal" manifolds and related partition lattices, Adv. Math. 110 (1995), no. 2, 277-313. MR MR1317619 (95m:52029) 
[9] E. Brieskorn, Die Fundamentalgruppe des Raumes der regulären Orbits einer endlichen komplexen Spiegelungsgruppe, Invent. Math. 12 (1971), 57-61. MR MR0293615 (45 \#2692)

[10] M. Davis, T. Januszkiewicz, and R. Scott, Nonpositive curvature of blow-ups, Selecta Math. (N.S.) 4 (1998), no. 4, 491-547. MR MR1668119 (2001f:53078)

[11] Pierre Deligne, Les immeubles des groupes de tresses généralisés, Invent. Math. 17 (1972), 273-302. MR MR0422673 (54 \#10659)

[12] Edward Fadell and Lee Neuwirth, Configuration spaces, Math. Scand. 10 (1962), 111-118. MR MR0141126 (25 \#4537)

[13] Eva Maria Feichtner and Dmitry N. Kozlov, On subspace arrangements of type D, Discrete Math. 210 (2000), no. 1-3, 27-54, Formal power series and algebraic combinatorics (Minneapolis, MN, 1996). MR MR1731606 (2001k:52039)

[14] R. Fox and L. Neuwirth, The braid groups, Math. Scand. 10 (1962), 119-126. MR MR0150755 (27 \#742)

[15] James E. Humphreys, Reflection groups and Coxeter groups, Cambridge Studies in Advanced Mathematics, vol. 29, Cambridge University Press, Cambridge, 1990. MR MR1066460 (92h:20002)

[16] Mikhail Khovanov, Real $K(\pi, 1)$ arrangements from finite root systems, Math. Res. Lett. 3 (1996), no. 2, 261-274. MR MR1386845 (97d:52023)

[17] Peter Orlik and Hiroaki Terao, Arrangements of hyperplanes, Grundlehren der Mathematischen Wissenschaften [Fundamental Principles of Mathematical Sciences], vol. 300, Springer-Verlag, Berlin, 1992. MR MR1217488 (94e:52014)

[18] Günter M. Ziegler, Lectures on polytopes, Graduate Texts in Mathematics, vol. 152, Springer-Verlag, New York, 1995. MR MR1311028 (96a:52011) 